\title{
The Role of Iterative Reconstruction Techniques in Cardiovascular CT
}

\author{
John W. Nance Jr. • U. Joseph Schoepf • \\ Ullrich Ebersberger
}

Published online: 14 September 2013

(C) Springer Science+Business Media New York 2013

\begin{abstract}
Iterative reconstruction (IR) techniques for cardiovascular computed tomography (CT) have enjoyed a resurgence of interest in recent years as computer power has increased enough to enable reasonably timely reconstructions. The major purported benefit of current IR techniques involves image noise reduction, which both provides improved image quality and enables radiation dose reductions. Several widely available products have been released by the major $\mathrm{CT}$ vendors that vary in their underlying techniques but, according to the current literature, give similar results. Future algorithms should both refine current IR techniques and expand the role of IR to additional cardiovascular CT applications. This review examines the technical basis of IR, the IR products available commercially, the current data on IR in cardiovascular $\mathrm{CT}$, and the future directions of the field.
\end{abstract}

Keywords Iterative reconstruction - Radiation dose Cardiac CT angiography · Image quality · Artifacts

J. W. Nance Jr. $(\bowtie)$

Department of Radiology and Radiological Science, The Johns Hopkins Hospital, Johns Hopkins University School of Medicine, 600 N. Wolfe Street, Baltimore, MD, USA

e-mail: jnance5@jhmi.edu

\section{U. J. Schoepf}

Heart and Vascular Center, Medical University of South Carolina, Charleston, SC, USA

U. Ebersberger

Department of Cardiology and Intensive Care Medicine, Heart Center Munich-Bogenhausen, Munich, Germany

\section{Introduction}

The development of computed tomography (CT) for medical imaging has had a profound impact on the practice of medicine over the past four decades. Technical advances in CT continue to develop at a remarkable pace. Nowhere is this more apparent than in cardiovascular CT. The inherent problems associated with imaging small, moving structures limited the role of cardiovascular CT for several decades. However, the past 20 years have seen a host of new developments that have brought cardiovascular CT into routine clinical practice. These began with the rise of cardiovascular electron beam CT in the mid 1990s and the subsequent development of multi detector-row CT and ECG-gating techniques just before the new millennium [1 2,3]. Clinicians and industry continue to promote hardware and software solutions with the fundamental objectives of improving image quality (e.g. improving temporal and spatial resolution; reducing artifacts), expanding clinical applications (e.g. myocardial perfusion imaging; left ventricular functional analysis), and reducing radiation dose. This review examines the current and future role of one such advance-iterative reconstruction (IR) techniques.

IR technology, although attracting much attention in CT applications in recent years, is not a recent development. Initial CT efforts in the early 1970s used IR techniques to create the first images from CT projectional data [4], and emission tomography has used iterative techniques since the 1960s [5]. IR, however, necessitates complex mathematical models and performs multiple reconstructions in the creation of images (see below), and, as CT resolution improved, increased computational demands rendered IR too slow for clinical use. Faster analytical algorithms, most prominently filtered back projection (FBP), became the dominant reconstruction methods, and IR was relegated to 
research applications. The renewed interest in IR comes as the exponential growth in computer power has started to meet the requirements for practical clinical use.

The current role of IR in cardiovascular CT is evolving. The fundamental technical advantage of IR is improved image quality, mainly via noise reduction but also as a result of artifact suppression. Several specific situations in particular may benefit, including examinations involving obese individuals, which are traditionally limited by high noise secondary to quantum mottle, and evaluation of coronary stents and heavily calcified vessels, which suffer from beam-hardening artifacts and suboptimum spatial resolution. Importantly, image noise is inversely related to radiation dose; therefore, improved noise characteristics have the potential to reduce the radiation dose associated with CT while retaining acceptable levels of image quality. The potential for significant dose reduction has been described as the main clinical advantage of IR, especially given current concerns from both the public and medical communities regarding radiation from diagnostic imaging [6]. In addition, researchers are currently developing new algorithms with the objectives of both improving the traditional strengths of IR and providing new advantages and applications of the technique.

\section{Technical Basis}

The mathematics underlying IR techniques are beyond the scope of this review. The reader is referred to Beister et al. [7] and similar articles for a more detailed technical discussion. Nevertheless, a conceptual understanding of IR techniques is necessary to appreciate their benefits, potential applications, and vendor-specific differences. The latter point deserves emphasis - "IR" is a fairly nonspecific term, and there are significant variations in the techniques, advantages, and disadvantages of the currently available commercial products.

\section{Basic Data Acquisition}

CT data are acquired by transmitting a fan beam of photons through the body to an array of detectors; to acquire a complete dataset this must be performed over multiple angles (at least $180^{\circ}$ ) around the long axis of the patient. Because the beam passes through the patient, the individual data points measured by each detector represent the sum of the attenuation of all tissues through which the beam has passed. All reconstruction methods create stacks of twodimensional (2D) images from this raw projection data. 2D image matrices are made up of an array of pixels, with each pixel corresponding to a specific area within the imaged subject and assigned an attenuation value. Differences between reconstruction techniques involve determining how this attenuation value is assigned in the final image.

Reconstruction with Traditional Analytical Methods

Traditional analytical reconstruction methods, for example FBP, gather all of the individual data points for a given detector element (the line integrals representing the total attenuation of the beam as it takes a radial path through the patient) and project that information back along the radial path, dividing the total attenuation evenly across all pixels in the path. This is repeated as the tube(s) and detectors rotate around the patient, and the combined attenuation values provided from each of these back projections are summed for each pixel, resulting in the final image. Mathematical filters are applied to the data before back projection to modify image noise and resolution. For simplicity, total available image noise reduction and spatial resolution can be regarded as fixed, and filters provide the optimum balance between resolution and noise for a given application (each at the expense of the other).

Analytical reconstruction methods are fast and fairly robust in many situations; however, there are multiple problems with the technique. In the most basic terms, analytical reconstructions use the measured signal as if all data were perfect. The photon beam is assumed to be a straight ray that arises from a single point on the surface of the anode, terminates at a point at the center of the detector, and interacts with a point in the center of each voxel. Realworld geometric considerations, such as focal spot size, anode heel effect, the three-dimensional (3D) interaction of the beam with the voxel, and the 2D interaction of the beam with the detector, are ignored. X-ray spectra are assumed to be monoenergetic, and nonlinear effects along the assumed ray, for example scatter and beam-hardening, are not considered. Perhaps most importantly, analytical reconstruction methods do not account for image noise that results from Poisson statistical variations in photon numbers across the image plane.

\section{IR}

The fundamental differences between analytical and IR can be described as follows: whereas analytical reconstruction methods perform a single reconstruction assuming all data are perfect, IR techniques perform repetitive reconstructions applying mathematical models to account for known imperfections in the projection data. Each "iteration" is slightly modified from the previous until a predefined criterion is met. As noted above, "IR" is a nonspecific term, and there is substantial variation in the technical details of commercially available IR products beyond the preceding generalization. The advantages and disadvantages of 
specific methods are largely dependent on the type and scale of mathematical modeling applied to iterations. Several basic classification schemes have been described that - while imperfect - may be useful. The most prevalent of these include classification based on:

1 the general type of algorithm used; and

2 the data (i.e. raw data or image data) used in the reconstruction process.

Most IR products in current clinical use apply models of photon counting statistics to reduce image noise: There are random variations in the number of photons striking the detector across the image plane, with the distribution of photon number across a given area described by Poisson statistics. Noise is represented by the relative standard deviation of photon number, which is inversely proportional to the number of photons striking the detector. Practically speaking, this explains why increasing tube current, which increases the number of X-rays transmitted, reduces image noise. By correlating the noise patterns with the signal (photon number) at the detector, statistical IR methods are able to selectively eliminate image noise with each successive iteration (Fig. 1). Statistics-based methods also may employ regularization techniques, the most common of which are "smoothing" algorithms that limit the allowable difference in attenuation between adjacent voxels.

More advanced "model-based" IR (MBIR) products go beyond statistical modeling. Both geometric (e.g. the area of the anode, interaction of the photon beam with the voxel and detector, anode heel effect, etc.) and physical (e.g. the X-ray spectra, scattered photons, beam-hardening, etc.) models can be applied. These models are used to predict the volumetric image, with the objective of approximating the actual image as closely as possible. The predicted image is forward-projected to create an artificial raw data set that is then compared with the actual raw data set. The predicted data are corrected on the basis of the actual data, and this correction is back projected to create an updated image. The process is repeated until a specific criterion is fulfilled-it can be performed for a fixed number of iterations, until the difference between the predicted and actual data reaches a predefined threshold, or until indicators of image quality reach a specified level [7].

IR methods may also be classified on the basis of the data that are reconstructed. IR performed only in the image/slice domain first reconstructs an image using FBP; this is then forward-projected using photon statistics to selectively remove noise across successive iterations. Techniques using both image and projection/raw data first reconstruct an image using FBP. Rather than directly modifying the real data as before, the actual raw data are compared with an artificial raw data set that was created by use of Poisson statistics-based noise models. Successive iterations again reduce image noise until the real and predicted data converge. Finally, IR techniques utilizing the projection data only are akin to the previously described MBIR methods. Complex statistical, geometric, and physical models attempt to predict the projection data. Predicted raw data are compared with actual raw data and modified over successive iterations [7, 8••].

The major advantage of statistical IR techniques involves noise reduction without a corresponding decrease in spatial resolution. The lower the signal to noise ratio (SNR), the more impact IR can have on image quality. Situations in which the SNR is inherently lower, for example evaluation of large patients and high-resolution acquisition utilizing small voxels (e.g. cardiac imaging), are particularly well-suited to IR applications (Fig. 2). The noise reduction properties of IR also enable reduction of radiation dose without unacceptable sacrifices in image quality. MBIR provides incremental improvements in image quality by reducing a variety of artifacts caused by invalid assumptions and physical and geometric imperfections in the transmission data. Streak artifact, beamhardening, motion, and scatter effects can be reduced.
Fig. 1 Standard FBP (a) and iterative (b) reconstructions at the same level of the ascending aorta. Image noise expressed as the standard deviation of the attenuation (HU) in the region of interest was significantly lower in images reconstructed using IR (SAFIRE; Siemens) (circle in b) than in those reconstructed using FBP (circle in a)
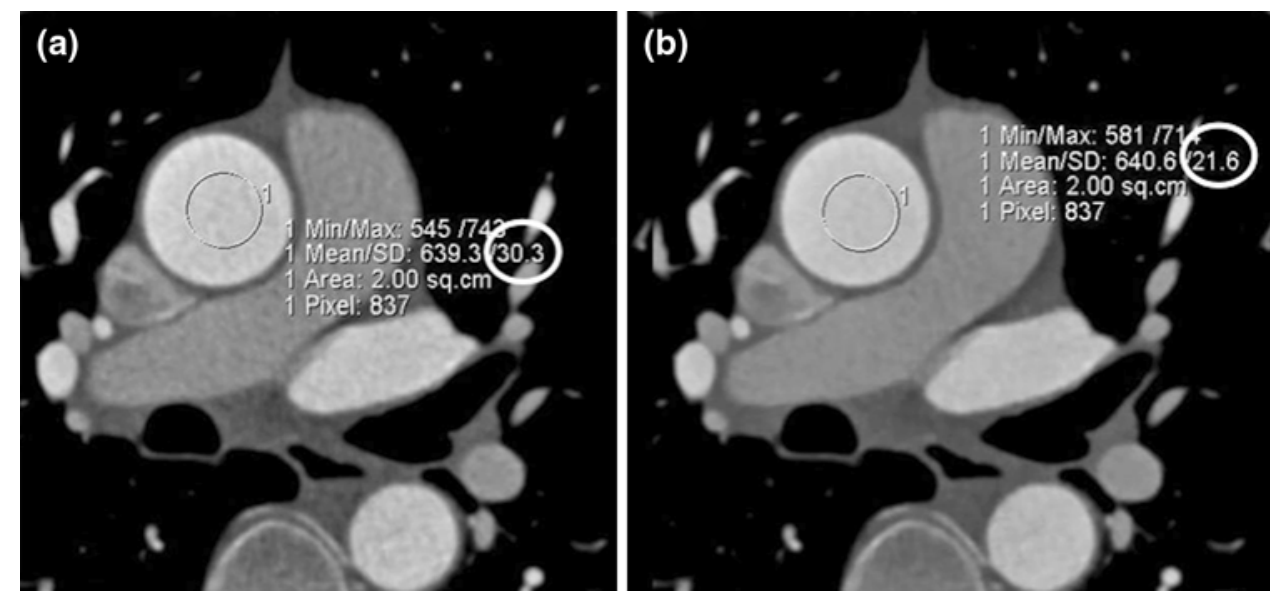
Fig. 2 Transverse a FBP and b IR (IRIS; Siemens) images at the level of the aortic root show extensive calcified plaque burden in the proximal left anterior descending coronary artery. Threshold-dependent volumetry of calcifications (purple) resulted in a measured volume of $61.34 \mathrm{~mm}^{3}$ on FBP reconstructions (c) and of $45.32 \mathrm{~mm}^{3}$ on IRs (d) (Color figure online)

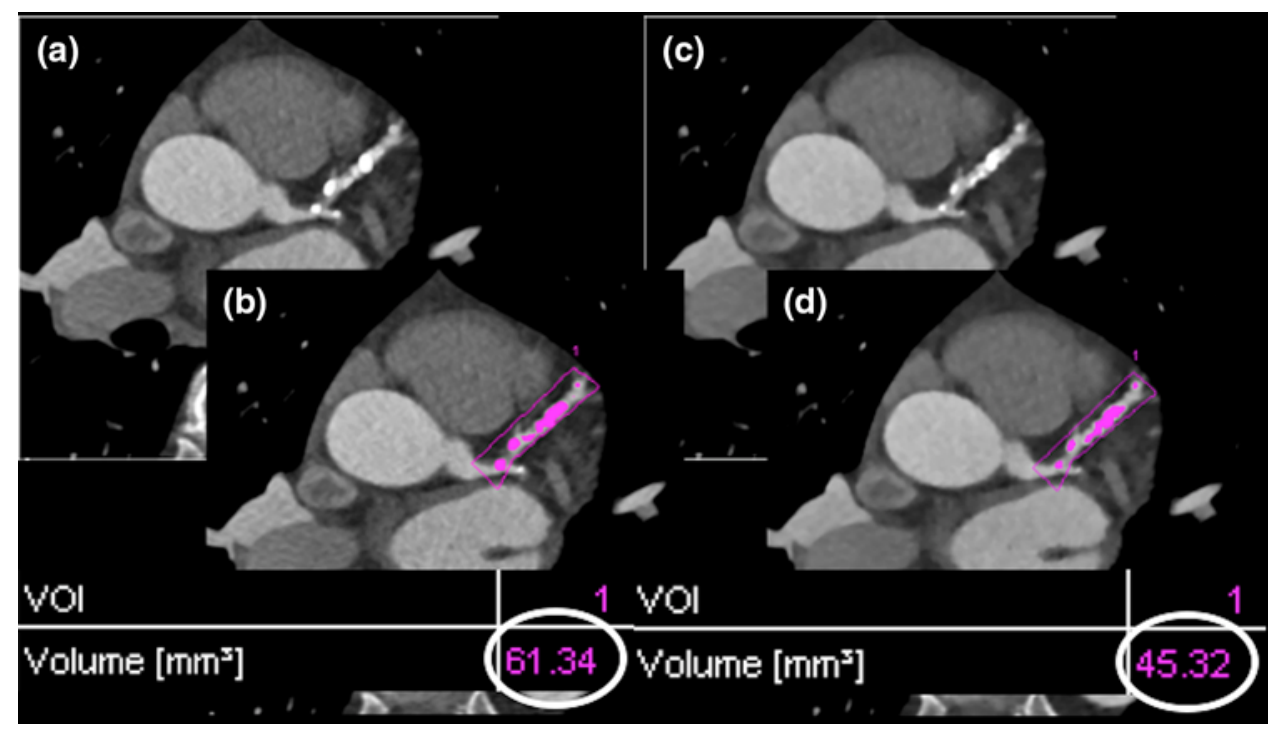

The main disadvantage of current IR techniques is the increased computational effort. Whereas this is negligible in most statistics-based methods, MBIR in the raw data domain can still take as long as 10-90 min per reconstruction [9]. Computational power continues to increase; however, the increased complexity of mathematical models and expansion of complex acquisition techniques, for example dualsource and dual-energy CT, have somewhat mitigated improvements in reconstruction time [7]. The quality of IR is also dependent on the robustness of the applied algorithms. Improper models and overcorrection can result in reduced image quality and novel artifacts. There is potential to lose information when data are manipulated in the projection domain. Finally, IR results in an alteration in the expected image appearance, which has been described as "waxy" or "plastic" [9]. The psychophysical and cognitive effects of this altered image appearance are difficult to quantify and may affect interpretation. Vendors have responded by providing different iteration "strengths" or enabling variable blending of IR with FBP images.

\section{Currently Available IR Products}

\section{Adaptive Statistical IR}

Adaptive statistical IR (ASIR; GE Healthcare, Waukesha, WI, USA) was the first modern CT IR product released, in 2008. For each axial image, ASIR creates an image noise map and a traditional FBP reconstruction. Attenuation variances between pixels in the FBP image are examined, and the ASIR algorithm identifies variances that are statistically unlikely, i.e. attenuation fluctuations more likely secondary to image noise than actual anatomic structures. The image noise map is then used to modify the FBP image on the basis of ideal noise modeling. This process is repeated until the modified FBP image and ideal pixel values converge. ASIR enables selective blending with FBP projections, with the most commonly reported blending ratios using 40-60\% ASIR [8••, 10] (Fig. 3).

IR in Image Space

IR in image space (IRIS; Siemens Healthcare, Forchheim, Germany) also uses statistics-based noise modeling; however, the entire process is carried out in the image domain. A nonlinear algorithm is applied to each area of an axial FBP image to remove attenuation variances that are likely to be because of noise. In general, a predefined and fixed number of iterations are performed [11] (Fig. 4).

\section{Sinogram Affirmed IR}

The second IR product released by Siemens, sinogram affirmed IR (SAFIRE), models noise on the basis of raw data. Attenuation variances are removed on the basis of this model and the resulting image is compared with, and combined with, the initial FBP image; the process is then repeated. SAFIRE most commonly uses five iterations; however, the number or "strength" of iterations can be modified [12] (Fig. 5).

\section{Adaptive Iterative Dose Reduction}

Adaptive iterative dose reduction (AIDR, Toshiba Medical Systems, Otawara, Japan) was the first IR method introduced by Toshiba; similar to IRIS, AIDR performs denoising of the image data on the basis of photon counting statistics. AIDR 3D was subsequently released with several major differences. Both electronic and quantum noise are 
Fig. 3 CT topography of an obese patient (a) with corresponding transverse reconstructions of a coronary CT angiography using FBP (b) and IR (IRIS; Siemens) (c). Image noise expressed as the standard deviation of the attenuation (HU) in the region of interest was significantly lower in images reconstructed using IR than in those reconstructed using $\mathrm{FBP}-18.9$ versus $39.3 \mathrm{HU}$

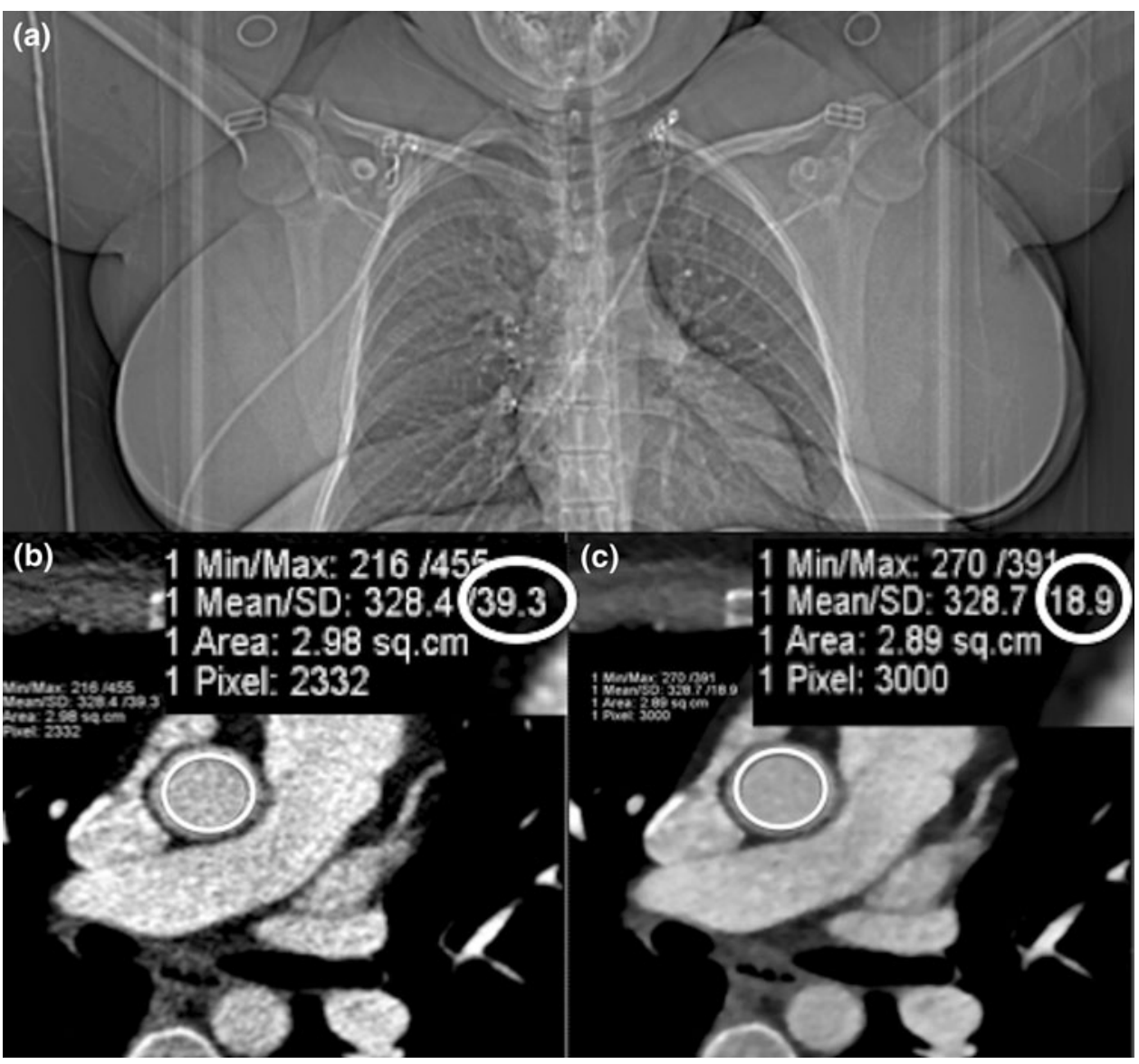

selectively removed in the projection domain, by both noise filtering and modeling. An additional model accounts for the physical properties of the CT system at the time of acquisition. These two processes are combined and subsequent iterations compare the original FBP and the modified reconstructions in the image domain. Anisotropic diffusion is used to ensure that high-frequency structures representing fine details are preserved $[13,14]$. Put simply, anisotropic diffusion models the shapes of the anatomic area being examined and provides shape-adaptive smoothing. For example, the system might detect high variation between two adjacent pixels within the left ventricular cavity. This is unexpected and would be attributed to noise and selectively eliminated. However, high variation between pixels at the myocardial-left ventricular border would be expected; this border would be left unchanged. Similar to other methods, iterations are repeated until a specific criterion is met. AIDR images are then blended with FBP images. Modifications of blending ratios are not available on current commercial products.

iDose

iDose, released by Philips Healthcare (Eindhoven, the Netherlands), can be compared with AIDR 3D in that it uses both noise and anatomic modeling in image reconstruction. First, the projection data are examined, areas with very low photon counts (i.e. noisy areas) are identified, and noise is selectively eliminated. Again, an anisotropic diffusion process is utilized to penalize noise while maintaining true anatomic edges. The process then moves to the image domain, where noise subtraction is performed using an estimated map of image noise so that edges are maintained. This "hybrid" (i.e. noise and anatomic modeling) method is purported to enable greater IR strength, resulting in less noise, without resulting in the waxy or overcorrected images that resulted from earlier generations of IR techniques. Seven different IR levels can be set (L1-L7) that correspond to increasing levels of noise reduction, ranging from a noise reduction factor of 0.89 (89\% noise compared with the equivalent FBP reconstruction) for L1 up to 0.45 for L7. These noise reduction factors are not arbitrary values; rather, they correspond to the noise increases that would result from increased levels of dose reduction, from $20 \%$ dose reduction for L1 to $80 \%$ reduction for L7 [15] (Fig. 1).

\section{MBIR/Veo}

MBIR, later released under the trade name Veo (GE Healthcare, Waukesha, WI, USA), is the only fully model- 
Fig. 4 Cardiac CT angiography (cCTA) study of a patient with an implanted coronary artery stent. Images are displayed as automatically generated curved multiplanar reformat along the vessel centerline (right figures; b and d) and as cross-sections perpendicular to the centerline (left figures; $\mathbf{a}$ and $\mathbf{c}$ ). The lower figures (c and $\mathbf{d}$ ) show images reconstructed using IR

(SAFIRE; Siemens) compared with those reconstructed using FBP (a and b)
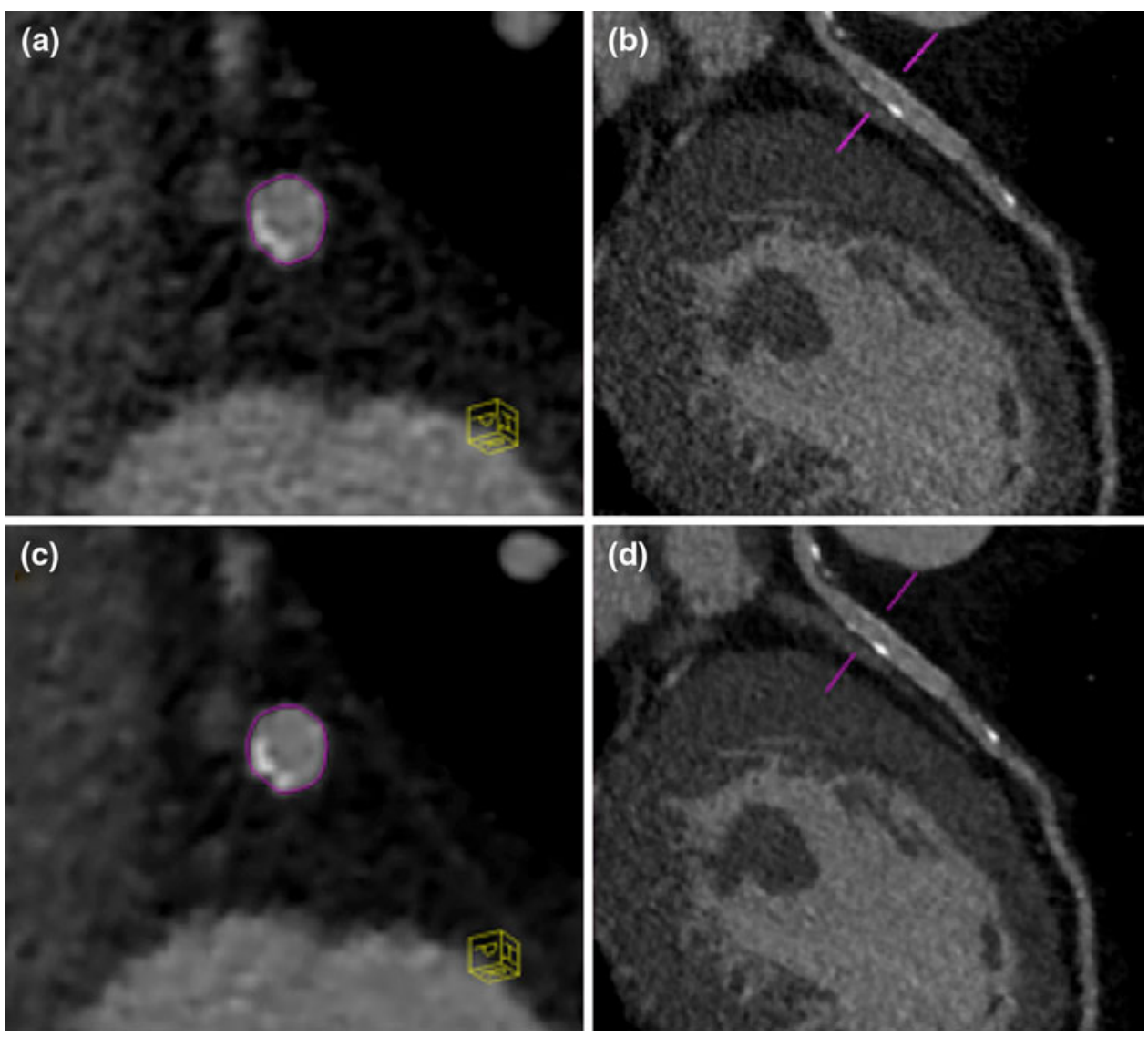

based reconstruction product currently available. It uses complex statistical, optical, geometric, and physical modeling that attempts to model the X-ray beam from anode to detector (see above). There are many potential benefits to the technique; but the complexity of the method is its major limitation, with reconstruction times ranging from 10 to $90 \min [9]$.

\section{Current Applications and Evidence}

Noise and Radiation Dose Reduction

The primary role of IR techniques in current cardiovascular CT practice is, via noise reduction, to enable radiation dose reduction without sacrificing image quality. Tube voltage and tube current are related to both radiation dose and image noise. Low voltage and/or low current acquisition procedures have become increasingly popular as dosereduction strategies, but at the expense of increased noise. Numerous studies have shown that IR can improve objective noise, SNR, contrast-to-noise ratio (CNR), and subjective image quality (SIQ) compared with FBP reconstructions of the same projection data; furthermore, low-dose acquisition using IR can maintain the image quality of higher-dose acquisition using FBP.
There are limitations in the currently available evidence. Most studies use objective and subjective measures of image quality; however, only limited data are available comparing the actual diagnostic accuracy of FBP and IR techniques. There are few studies that compare specific IR products with each other. Most IR applications have modifiable settings involving the iterative strength or proportion of IR blending with FBP; however, the optimum settings for each product and situation are not well-defined. There are limited data on the impact of IR on clinical workflow. Finally, the radiation dose reductions reported in many studies are simulated or estimated (e.g. by removing half of the projection data or adding artificial noise to the images) rather than actual.

With these limitations in mind, the current evidence shows that IR techniques may have extraordinary dosereduction potential in a variety of clinical scenarios. Selected studies are summarized in Table 1. We can summarize the trends as follows: IR results in reduced noise with no to minimal effects on attenuation (signal) compared with FBP reconstructions of the same data, resulting in improved SNR and CNR. This usually, but not uniformly, results in improvements in SIQ and vessel assessment. In general, noise reduction is proportional to the iterative strength or proportion of IR blending; however, most studies reveal that SIQ does not follow the same 
Fig. 5 cCTA study of a 70 year old patient (BMI 24) using a prospective gated scan and ASiR (Adaptive Statistical IR; GE) image reconstruction. Despite a dose-lengthy product (DLP) of only $37 \mathrm{mGy}-\mathrm{cm}$ (0.5 mSV), excellent image quality was achieved. a, d Curved multiplanar reformation of the left anterior descending coronary artery (LAD) and the right coronary artery (RCA). Black arrows indicate severe lumen narrowing caused by mixedplaques (a) and noncalcified plaque (d). b 3D volume rendering from left anterior oblique perspective c Corresponding virtual angioscopy with severe calcified lesions (black arrow). (Images courtesy of GE Health Care.)

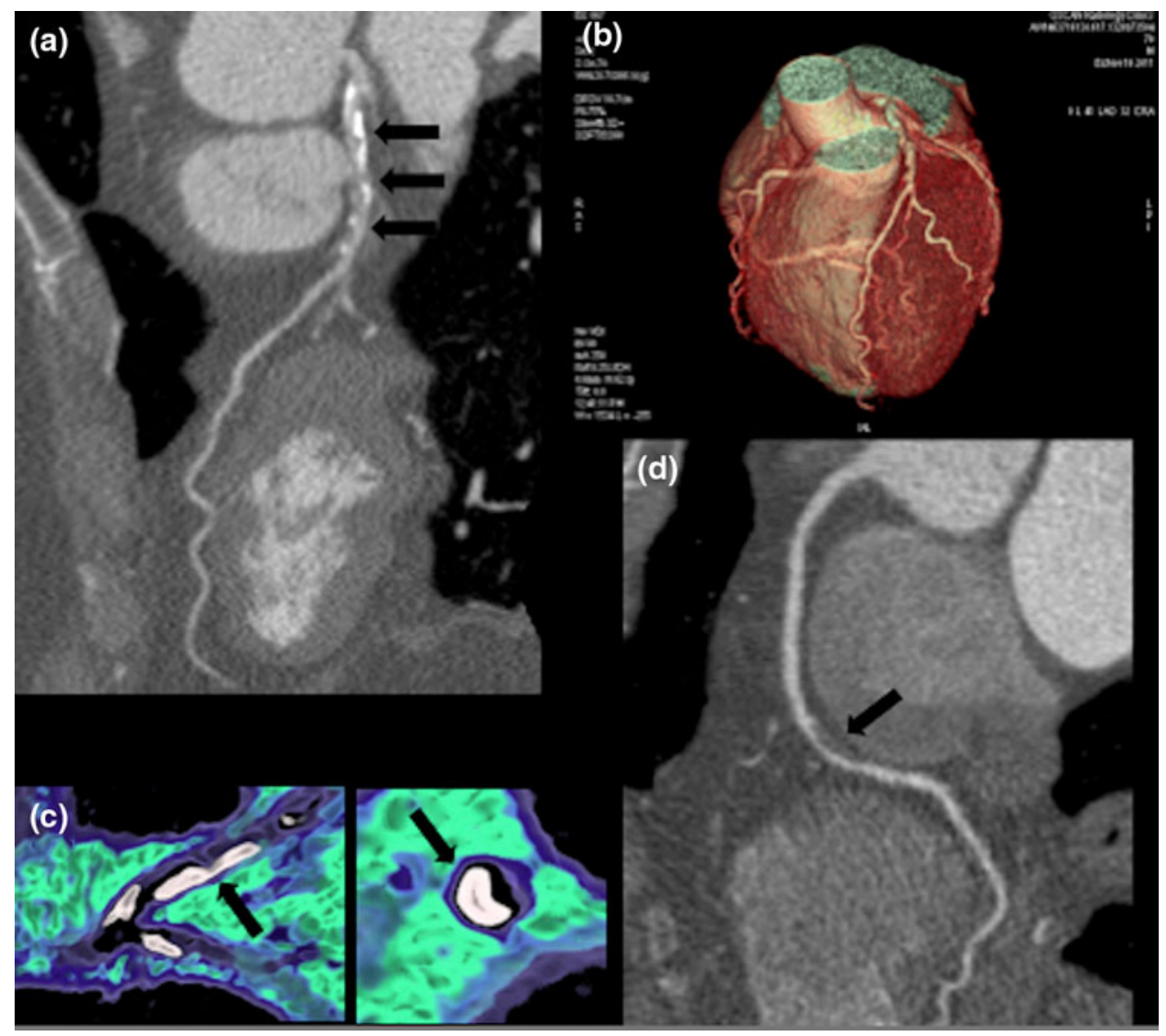

pattern. As iterative strength/proportion increases, SIQ first improves, peaks, but then begins to diminish, because of overcorrection and/or the introduction of new artifacts, and/or an increasingly unfamiliar subjective image appearance may evolve. Most studies that compare actual or simulated low-dose acquisitions reconstructed with IR to higher-dose acquisitions reconstructed with FBP demonstrated no significant differences or persistent improvements in SIQ with the use of IR [12, 14, 16-26, 27•, 2833]. Several real-world observational studies have recorded cardiovascular CT dosimetry data after implementation of IR methods and likewise shown significant dose reductions without unacceptable sacrifices in SIQ [18, 31, 34•]. The few studies that have assessed the diagnostic accuracy of IR using intravascular ultrasound (for coronary artery plaque characterization) or invasive coronary angiography (for coronary artery stenosis detection and quantification) have reported equivalent or improved accuracy compared with FBP [12, 25, 26, 35, 36].

These findings are valid for all currently available IR products except MBIR, for which experiences are extremely limited. ASIR has resulted in improved image quality at a dose reduction of $25 \%$ compared with FBP [37] and equivalent image quality and diagnostic accuracy with dose reductions up to $72 \%$ [25]. Observational studies after implementation of ASIR have demonstrated 44-54\% reductions in effective dose [18, 34•]. AIDR has shown improved subjective and objective image quality with simulated $50 \%$ dose reduction compared with full-dose FBP reconstructions of the same data [38], and one observational study demonstrated dose savings of $22 \%$ without compromising quality [31]. Low-dose acquisitions using IRIS have demonstrated improved image quality compared with routine acquisitions using FBP with dose savings up to $62 \%$ [27•]. Likewise, SAFIRE has shown equivalent image quality as FBP at simulated dose reductions of $50 \%$ [39] and improved image quality with simulated dose reductions of 50-80\% [12, 29]. Both fixed and adaptive-dose acquisition procedures using iDOSE have shown that radiation reductions of 55-63\% are allowable with improved or equivalent image quality compared with higher-dose FBP [15, 20, 40]. Preliminary investigations using MBIR have shown favorable data on diagnostic accuracy [36], demonstrating improved image quality compared with ASIR in one study [41]; however, the evidence for MBIR in cardiovascular CT is currently limited to ex-vivo studies.

The optimum method for dose reduction using IR is not well-defined. Most of the more impressive dose reductions were demonstrated in simulated or fixed-dose reductions 


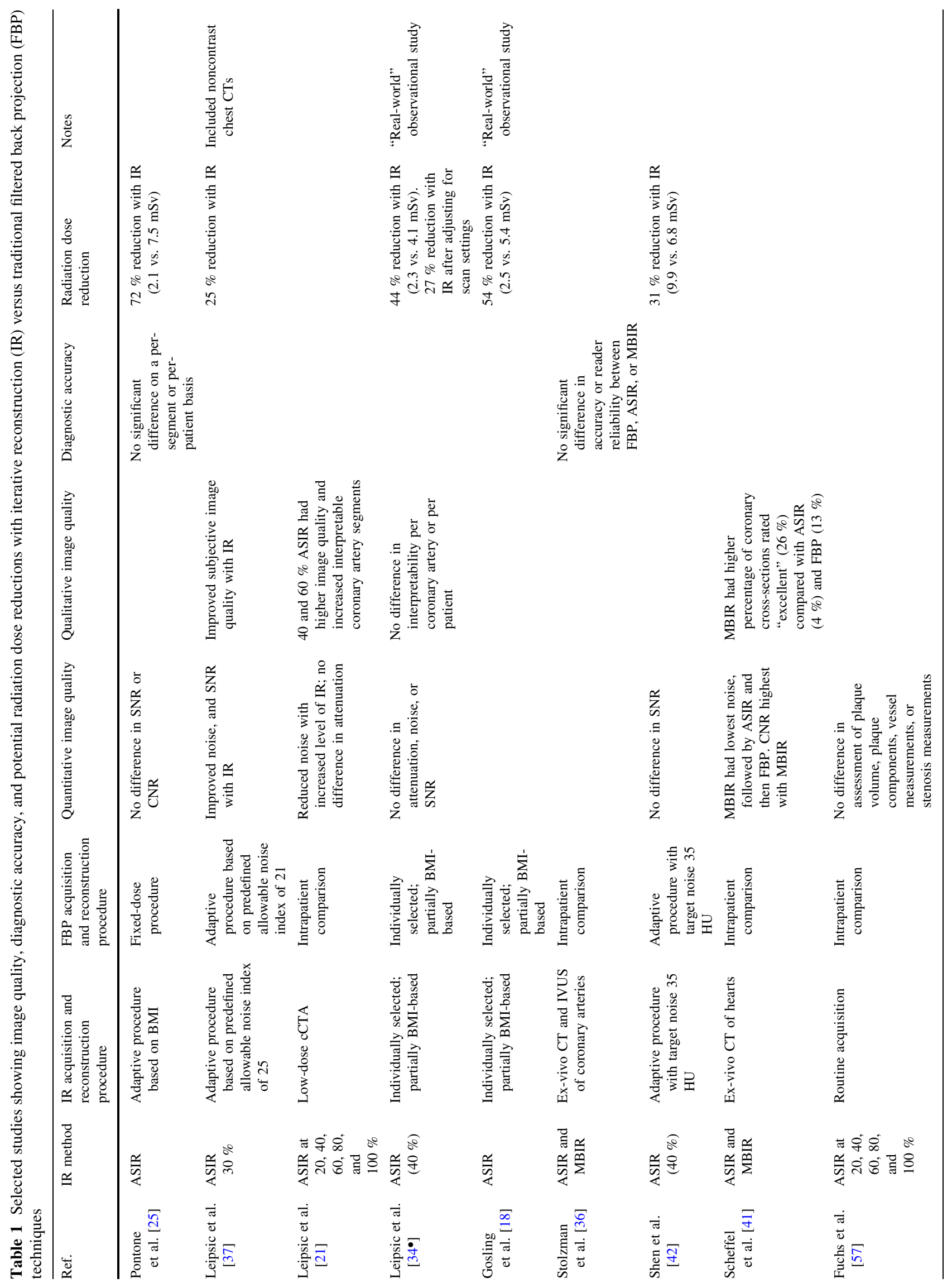




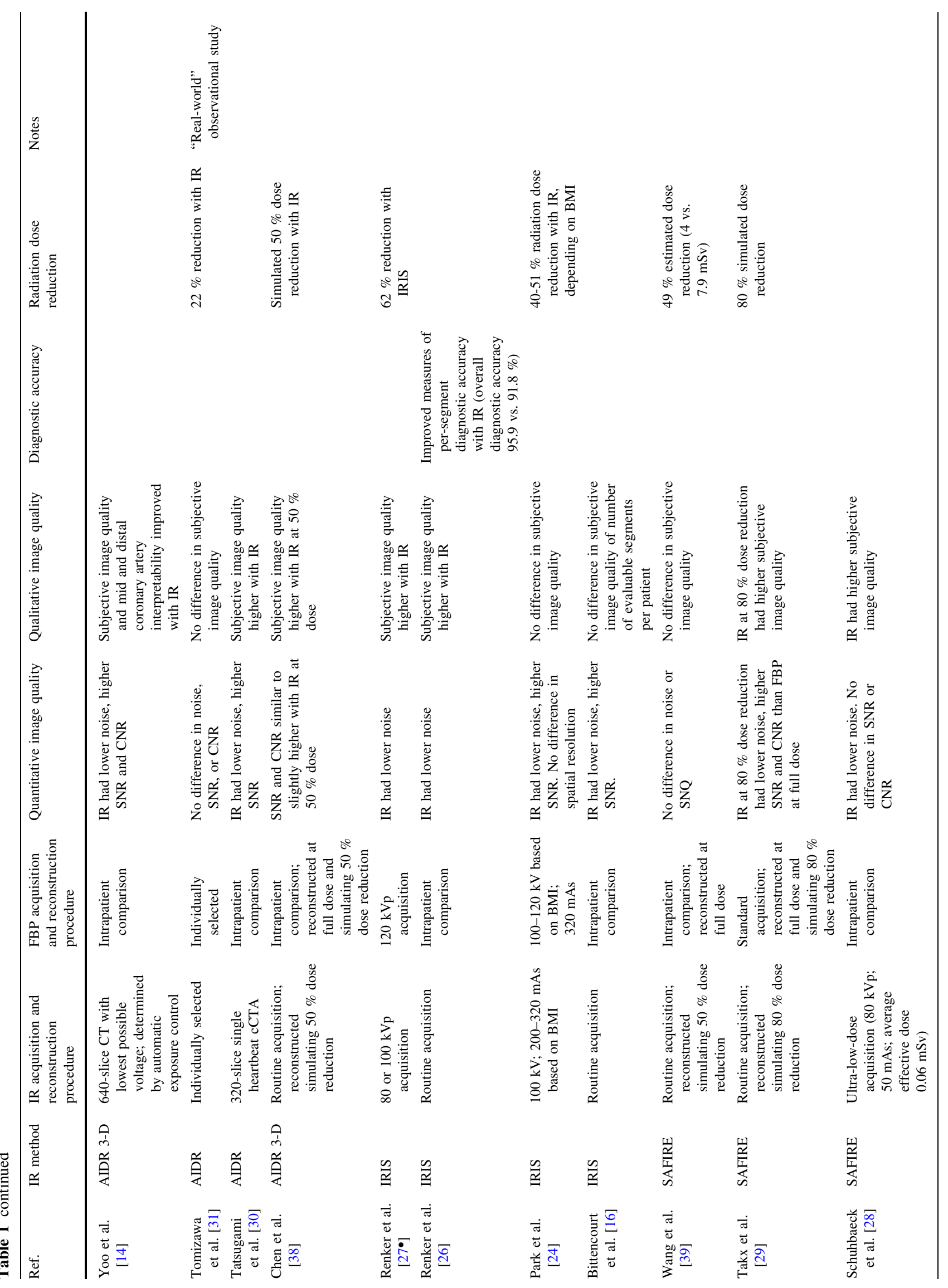




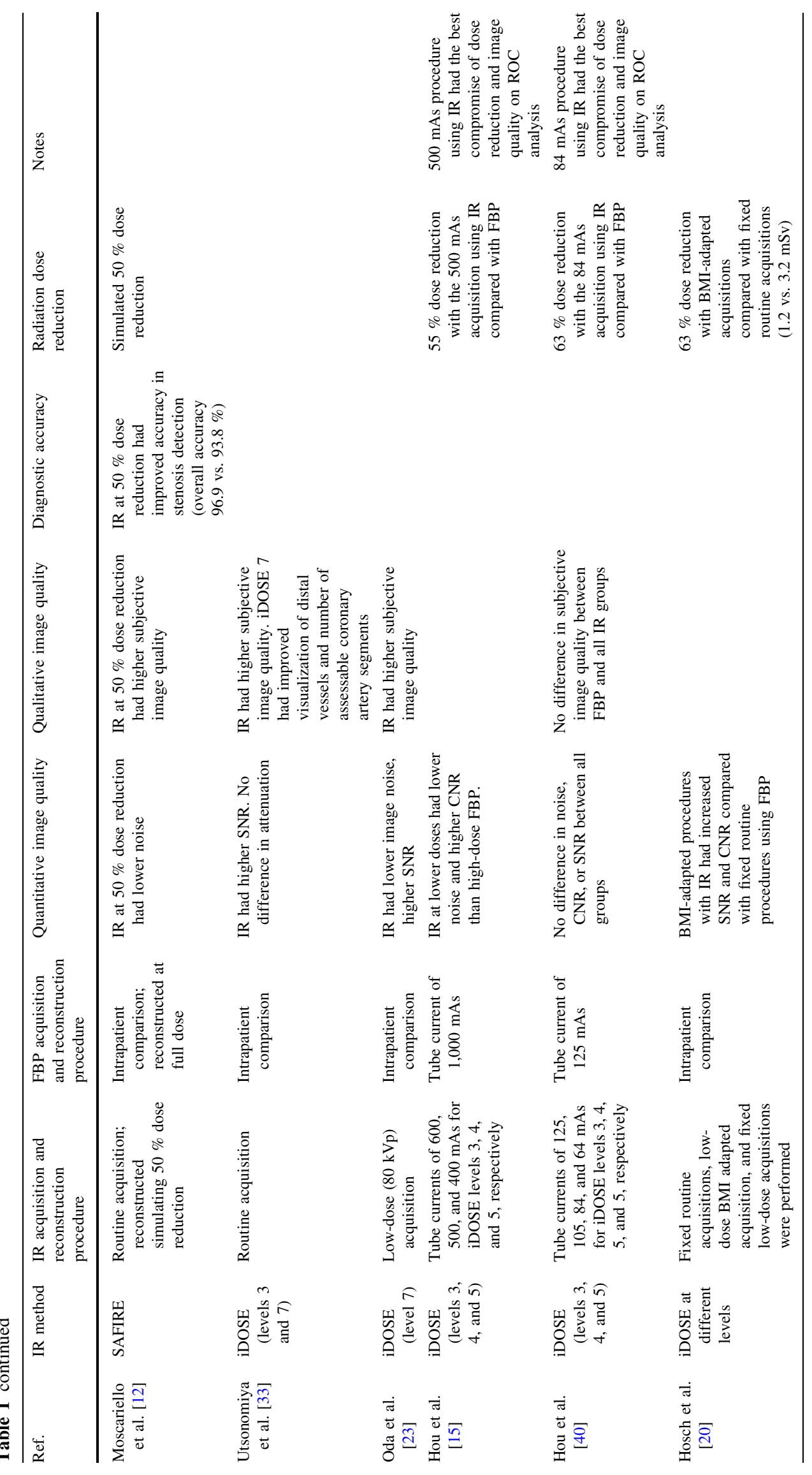


that may not be valid in clinical practice or have limitations in generalization to a broader population. BMI-adaptive reconstructions using predefined acquisition settings based on patient body habitus offer a potential solution [24, 25]; other groups have proposed patient-specific adaptive-dose procedures that adjust scan settings on the basis of allowable image noise [37, 42]. Notably, specific IR products vary in their patient-specific adaptability.

The use of IR methods has also been examined specifically in pediatric populations, with similar results. Both SAFIRE and IRIS have shown improvements in image noise, CNR, SNR, and SIQ compared with equivalent-dose FBP reconstructions [19, 32]. Simulated half-dose IRIS and SAFIRE reconstructions in pediatric cardiovascular CT compare favorably with full-dose FBP reconstructions, with SAFIRE showing the greatest benefit [32].

The noise-reduction properties of IR may hold particular appeal in the evaluation of obese individuals, in whom noise is intrinsically higher secondary to reduced photon transmission. One study demonstrated a $50 \%$ reduction in mean effective radiation dose using cardiovascular $\mathrm{CT}$ procedures with $100 \mathrm{kV}$ tube current and SAFIRE reconstructions compared with $120 \mathrm{kV}$ procedures reconstructed with FBP, without detrimental effects on image noise, SNR, CNR, or SIQ. The patient population was limited to individuals with BMI $>30 \mathrm{~kg} / \mathrm{m}^{2}$ [43]. Similar results have been reported for use of ASIR for obese individuals undergoing CT examinations of the abdomen and pelvis [44].
Fig. 6 Transverse a FBP, and IRs using iDose (Philips Healthcare) at iDose level b 4 and $\mathbf{c} 7$, at the level of the aortic root. A continuous decrease of image noise can be seen from images a through c. d Shows a $3 \mathrm{D}$ volume rendered image reconstructed using iDose level 7. (Images courtesy of Armin Huber, Department of

Radiology, Klinikum rechts der Isar, Technische Universität München, Munich, Germany.)
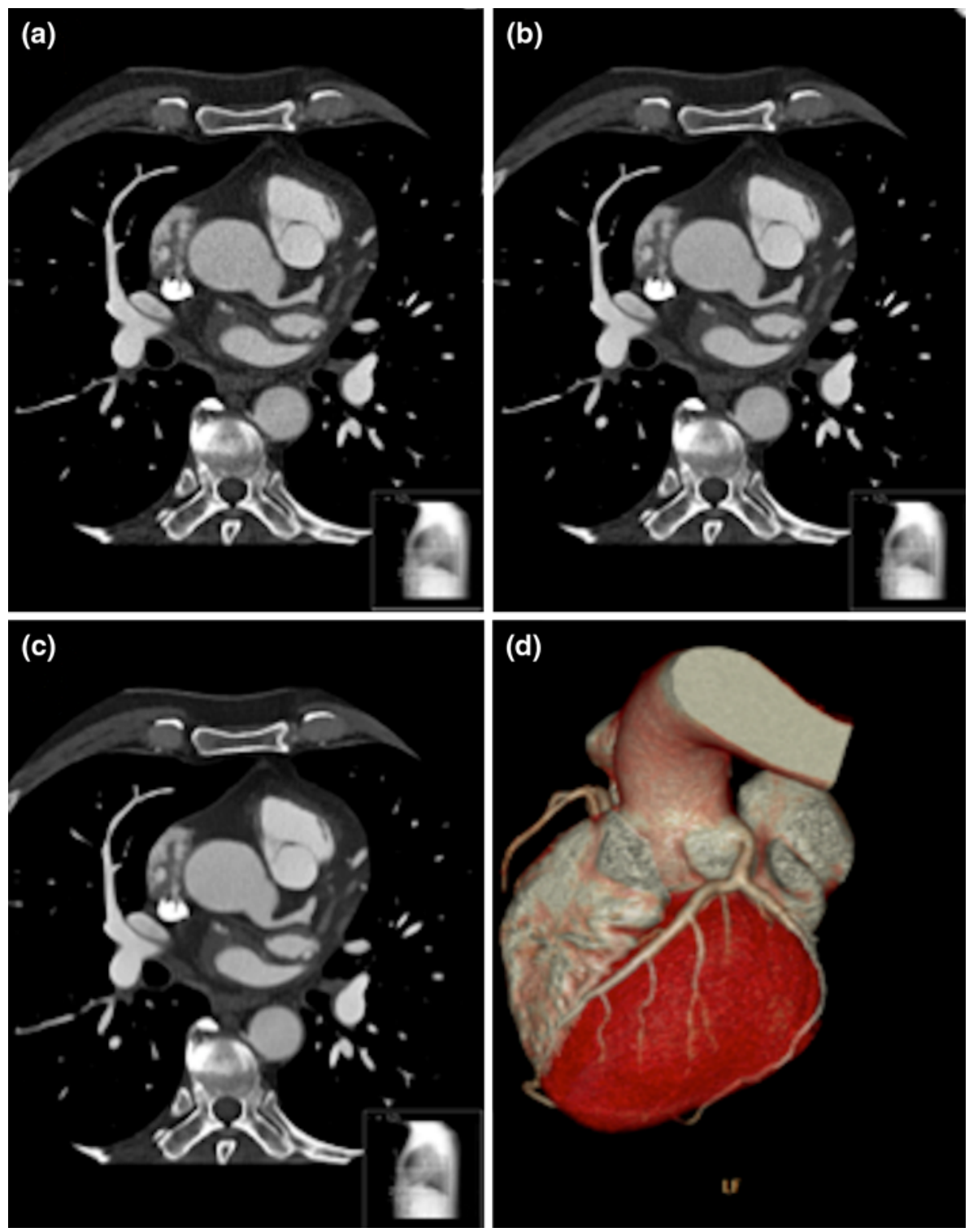
Coronary Artery Stent Evaluation

Evaluation of coronary artery stents using cardiovascular CT has been vigorously investigated but remains a challenge because of limitations in temporal resolution, spatial resolution, and beam-hardening artifacts [45]. Recent work has examined several potential roles of IR in improving CT stent evaluation. Studies simply comparing IR to FBP reconstructions for patients with coronary artery stents have demonstrated improvements in image quality with dose reductions similar to those described above. Reductions in stent volumes [27•, 46] and image noise [27•, 46-48] have been reported, with improved in-stent visualization [46, 48] (Fig. 6). IR may also provide value in high-resolution CT stent imaging. Spatial resolution is a major limitation in stent evaluation, prompting the development of high-resolution acquisition (usually $0.23 \mathrm{~mm}$ spatial resolution). Reduced voxel size in these images leads to significant problems with image noise because of photon starvation; this provides the basis for IR implementation. Early studies have shown improvements in noise, blooming artifacts, in-stent visualization, and diagnostic accuracy using IR in conjunction with high-resolution reconstruction kernels and acquisitions [49-51]. Custom-built IR algorithms that specifically target blooming artifacts may provide yet another solution for CT stent evaluations [52].

\section{Coronary Calcium Evaluation}

Similar concepts provide the basis for IR use in heavily calcified vessels. Renker et al. [26] demonstrated significantly lower image noise and calcification volumes with significantly higher SIQ when using IRIS compared with FBP for patients with Agatston scores $\geq 400$ (Fig. 4). IR also led to significant improvements in several measures of per-segment diagnostic accuracy for detection of significant stenoses, with overall diagnostic accuracy of $95.9 \%$ for IRIS compared with $91.8 \%$ for FBP, using invasive coronary angiography as reference standard. Reductions in calcification volume using IR should be considered when performing noncontrast cardiovascular CT for calcium scoring; one study comparing ASIR to FBP in CT calcium scoring showed decreased noise and reduced Agatston and volumetric calcium scores when using IR compared with FBP [53]. Because calcium score risk stratification uses data from previous population-based studies that used FBP reconstructions, coronary calcium volume reduction with IR has the potential to result in diverging risk stratification and subsequent aggressiveness of risk factor modification.

\section{Future Directions}

Short-term objectives for IR should include refinement of current techniques and applications. As noted above, the optimum strategy for IR-based radiation dose reductions is not established. BMI-adaptive [24, 25] and noise-adaptive [37, 42] acquisition procedures have been proposed. In addition, several IR products enable user-specified modulation of iterative strength or proportion of blending with FBP images, and researchers should seek to evaluate the best combinations for routine clinical work. Finally, more data regarding the effects of IR on diagnostic accuracy, prognostic value, and clinical outcomes will be necessary to justify widespread clinical adoption.

Early studies have shown that IR may provide cardiovascular CT solutions beyond traditional noise and dose reductions. High-resolution acquisitions are becoming more feasible as IR tempers the negative effects of photon starvation [49-51]. Contrast material dose reductions may also be possible without prohibitive degradation of image quality [22]. Novel IR algorithms are also emerging that should expand IR applications. Beam-hardening correction algorithms have been proposed for use in cardiovascular CT myocardial perfusion imaging to reduce artifacts related to high-concentration contrast material in the descending aorta and left ventricle [54]. Preliminary data on an IR technique that imposes penalties on dense tissues has likewise suggested a role for IR in reducing blooming artifacts [52], while a novel "metal detection technique" described by Boas and Fleischmann [55] demonstrated reduced beam-hardening artifacts from metal and bone. Future algorithms can be expected to further incorporate system, geometric, and motion models $[8 \bullet \bullet, 56]$.

\section{Conclusion}

Increases in available and affordable computer power have provided the basis for (re)application of IR to cardiovascular CT. Current data suggest that commercially available IR products provide noise reduction that enables significant reduction in radiation dose without degrading image quality. Refinement of current IR techniques should stimulate increased clinical adoption, and emerging and future algorithms promise to expand the role of IR to novel cardiovascular CT applications.

\section{Compliance with Ethics Guidelines}

Conflict of Interest John W. Nance Jr and Ullrich Ebersberger declare that they have no conflict of interest. U. Joseph Schoepf receives honoraria from and is a paid consultant for Bayer and Siemens and receives payment for development of educational presentations from GE. 
Human and Animal Rights and Informed Consent This article does not contain any studies with human or animal subjects performed by any of the authors.

\section{References}

Papers of particular interest, published recently, have been highlighted as:

- Of importance.

-• Of significant importance

1. Achenbach S, Kondo T. Technical advances in cardiac CT. Cardiol Clin. 2012;30:1-8. Well written overview of the progress in CT hardware technology and CT image reconstruction/processing software.

2. Flohr TG, Schoepf UJ, Ohnesorge BM. Chasing the heart: new developments for cardiac CT. J Thorac Imaging. 2007;22:4-16.

3. Ohnesorge BM, Hofmann LK, Flohr TG, Schoepf UJ. CT for imaging coronary artery disease: defining the paradigm for its application. Int J Cardiovasc Imaging. 2005;21:85-104.

4. Hounsfield GN. Computerized transverse axial scanning (tomography). 1. Description of system. Br J Radiol. 1973;46:1016-22.

5. Brooks RA, Di Chiro G. Principles of computer assisted tomography (CAT) in radiographic and radioisotopic imaging. Phys Med Biol. 1976;21:689-732.

6. Smith-Bindman R. Is computed tomography safe? N Engl J Med. 2010;363:1-4.

7. Beister M, Kolditz D, Kalender WA. Iterative reconstruction methods in X-ray CT. Phys Med Biol. 2012;28:94-108.

8. •- Leipsic J, Heilbron BG, Hague C. Iterative reconstruction for coronary CT angiography: finding its way. Int $\mathrm{J}$ Cardiovasc Imaging. 2012;28:613-20. Review of various iterative reconstruction platforms released for use for CCTA and initial experiences implementing and integrating these reconstruction algorithms in clinical practice.

9. Nelson RC, Feuerlein S, Boll DT. New iterative reconstruction techniques for cardiovascular computed tomography: how do they work, and what are the advantages and disadvantages? J Cardiovasc Comput Tomogr. 2011;5:286-92.

10. Marin D, Nelson RC, Schindera ST, et al. Low-tube-voltage, high-tube-current multidetector abdominal CT: improved image quality and decreased radiation dose with adaptive statistical iterative reconstruction algorithm-initial clinical experience. Radiology. 2010;254:145-53.

11. Pontana F, Pagniez J, Flohr T, et al. Chest computed tomography using iterative reconstruction vs filtered back projection (Part 1): evaluation of image noise reduction in 32 patients. Eur Radiol. 2011;21:627-35.

12. Moscariello A, Takx RA, Schoepf UJ, et al. Coronary CT angiography: image quality, diagnostic accuracy, and potential for radiation dose reduction using a novel iterative image reconstruction technique-comparison with traditional filtered back projection. Eur Radiol. 2011;21:2130-8.

13. Yamada Y, Jinzaki M, Hosokawa T, et al. Dose reduction in chest $\mathrm{CT}$ : comparison of the adaptive iterative dose reduction $3 \mathrm{D}$, adaptive iterative dose reduction, and filtered back projection reconstruction techniques. Eur J Radiol. 2012;81:4185-95.

14. Yoo RE, Park EA, Lee W, et al. Image quality of adaptive iterative dose reduction 3D of coronary CT angiography of 640-slice CT: comparison with filtered back-projection. Int J Cardiovasc Imaging. 2012;29(3):669-76.
15. Hou Y, Liu X, Xv S, et al. Comparisons of image quality and radiation dose between iterative reconstruction and filtered back projection reconstruction algorithms in 256-MDCT coronary angiography. AJR Am J Roentgenol. 2012;199:588-94.

16. Bittencourt MS, Schmidt B, Seltmann M, et al. Iterative reconstruction in image space (IRIS) in cardiac computed tomography: initial experience. Int J Cardiovasc Imaging. 2011;27:1081-7.

17. Gervaise A, Osemont B, Lecocq S, et al. CT image quality improvement using adaptive iterative dose reduction with widevolume acquisition on 320-detector CT. Eur Radiol. 2012;22: 295-301.

18. Gosling O, Loader R, Venables $\mathrm{P}$, et al. A comparison of radiation doses between state-of-the-art multislice CT coronary angiography with iterative reconstruction, multislice CT coronary angiography with standard filtered back-projection and invasive diagnostic coronary angiography. Heart. 2010;96:922-6.

19. Han BK, Grant KL, Garberich R, et al. Assessment of an iterative reconstruction algorithm (SAFIRE) on image quality in pediatric cardiac CT datasets. J Cardiovasc Comput Tomogr. 2012;6:200-4.

20. Hosch W, Stiller W, Mueller D, et al. Reduction of radiation exposure and improvement of image quality with BMI-adapted prospective cardiac computed tomography and iterative reconstruction. Eur J Radiol. 2012;81:3568-76.

21. Leipsic J, Labounty TM, Heilbron B, et al. Adaptive statistical iterative reconstruction: assessment of image noise and image quality in coronary CT angiography. AJR Am J Roentgenol. 2010;195:649-54.

22. Nakaura T, Kidoh M, Sakaino N, et al. Low contrast- and low radiation dose protocol for cardiac $\mathrm{CT}$ of thin adults at 256-row CT: usefulness of low tube voltage scans and the hybrid iterative reconstruction algorithm. Int J Cardiovasc Imaging. 2012;29(4): 913-23.

23. Oda S, Utsunomiya D, Funama Y, et al. A hybrid iterative reconstruction algorithm that improves the image quality of lowtube-voltage coronary CT angiography. AJR Am J Roentgenol. 2012;198:1126-31.

24. Park EA, Lee W, Kim KW, et al. Iterative reconstruction of dualsource coronary CT angiography: assessment of image quality and radiation dose. Int J Cardiovasc Imaging. 2012;28:1775-86.

25. Pontone G, Andreini D, Bartorelli AL, et al. Feasibility and diagnostic accuracy of a low radiation exposure protocol for prospective ECG-triggering coronary MDCT angiography. Clin Radiol. 2012;67:207-15.

26. Renker M, Nance JW Jr, Schoepf UJ, et al. Evaluation of heavily calcified vessels with coronary CT angiography: comparison of iterative and filtered back projection image reconstruction. Radiology. 2011;260:390-9.

27. - Renker M, Ramachandra A, Schoepf UJ, et al. Iterative image reconstruction techniques: applications for cardiac CT. J Cardiovasc Comput Tomogr. 2011;5:225-30. Well written manuscript suggesting that IR (IRIS) incrementally improves CT evaluation of coronary arteries, especially in challenging scenarios as severe calcifications. In addition it was shown that dose reduction can be achieved.

28. Schuhbaeck A, Achenbach S, Layritz C, et al. Image quality of ultra-low radiation exposure coronary $\mathrm{CT}$ angiography with an effective dose $<0.1 \mathrm{mSv}$ using high-pitch spiral acquisition and raw data-based iterative reconstruction. Eur Radiol. 2012;23: 597-606.

29. Takx RA, Schoepf UJ, Moscariello A, et al. Coronary CT angiography: comparison of a novel iterative reconstruction with filtered back projection for reconstruction of low-dose CT-Initial experience. Eur J Radiol. 2013;82:275-80.

30. Tatsugami F, Matsuki M, Nakai G, et al. The effect of adaptive iterative dose reduction on image quality in 320-detector row CT coronary angiography. Br J Radiol. 2012;85:378-82. 
31. Tomizawa N, Nojo T, Akahane M, et al. Adaptiveiterative dose reduction in coronary CT angiography using 320-row CT: assessment of radiation dose reduction and image quality. J Cardiovasc Comput Tomogr. 2012;6:318-24.

32. Tricarico F, Hlavacek AM, Schoepf UJ, et al. Cardiovascular CT angiography in neonates and children: image quality and potential for radiation dose reduction with iterative image reconstruction techniques. Eur Radiol. 2012;23:1306-15.

33. Utsunomiya D, Weigold WG, Weissman G, et al. Effect of hybrid iterative reconstruction technique on quantitative and qualitative image analysis at 256-slice prospective gating cardiac CT. Eur Radiol. 2012;22:1287-94.

34. • Leipsic J, Labounty TM, Heilbron B, et al. Estimated radiation dose reduction using adaptive statistical iterative reconstruction in coronary CT angiography: the ERASIR study. AJR Am J Roentgenol. 2010;195:655-60. Multi-center study including 574 patients showing lower radiation dose in comparison with FBP, with preserved signal, noise, and study interpretability.

35. Maffei E, Martini C, Rossi A, et al. Diagnostic accuracy of second-generation dual-source computed tomography coronary angiography with iterative reconstructions: a real-world experience. Radiol Med. 2012;117:725-38.

36. Stolzmann P, Schlett CL, Maurovich-Horvat P, et al. Variability and accuracy of coronary CT angiography including use of iterative reconstruction algorithms for plaque burden assessment as compared with intravascular ultrasound-an ex vivo study. Eur Radiol. 2012;22:2067-75.

37. Leipsic J, Nguyen G, Brown J, et al. A prospective evaluation of dose reduction and image quality in chest $\mathrm{CT}$ using adaptive statistical iterative reconstruction. AJR Am J Roentgenol. 2010; 195:1095-9.

38. Chen MY, Steigner ML, Leung SW, et al. Simulated 50\% radiation dose reduction in coronary $\mathrm{CT}$ angiography using adaptive iterative dose reduction in three-dimensions (AIDR3D). Int $\mathrm{J}$ Cardiovasc Imaging. 2013;29:1167-75.

39. Wang R, Schoepf UJ, Wu R, et al. CT coronary angiography: image quality with sinogram-affirmed iterative reconstruction compared with filtered back-projection. Clin Radiol. 2012;68: $272-8$.

40. Hou Y, Xu S, Guo W, et al. The optimal dose reduction level using iterative reconstruction with prospective ECG-triggered coronary CTA using 256-slice MDCT. Eur J Radiol. 2012;81: 3905-11.

41. Scheffel H, Stolzmann P, Schlett CL, et al. Coronary artery plaques: cardiac CT with model-based and adaptive-statistical iterative reconstruction technique. Eur J Radiol. 2012;81:363-9.

42. Shen J, Du X, Guo D, et al. Noise-based tube current reduction method with iterative reconstruction for reduction of radiation exposure in coronary CT angiography. Eur J Radiol. 2013;82: 349-55.

43. Wang R, Schoepf UJ, Wu R, et al. Image quality and radiation dose of low dose coronary CT angiography in obese patients: sinogram affirmed iterative reconstruction versus filtered back projection. Eur J Radiol. 2012;81:3141-5.
44. Desai GS, Uppot RN, Yu EW, et al. Impact of iterative reconstruction on image quality and radiation dose in multidetector CT of large body size adults. Eur Radiol. 2012;22:1631-40.

45. Sun Z, Almutairi AM. Diagnostic accuracy of 64 multislice CT angiography in the assessment of coronary in-stent restenosis: a meta-analysis. Eur J Radiol. 2010;73:266-73.

46. Ebersberger U, Tricarico F, Schoepf UJ, et al. CT evaluation of coronary artery stents with iterative image reconstruction: improvements in image quality and potential for radiation dose reduction. Eur Radiol. 2013;23:125-32.

47. Eisentopf J, Achenbach S, Ulzheimer S, et al. Low-dose dualsource CT angiography with iterative reconstruction for coronary artery stent evaluation. JACC Cardiovasc Imaging. 2013;6: 458-65.

48. Gebhard C, Fiechter M, Fuchs TA, et al. Coronary artery stents: influence of adaptive statistical iterative reconstruction on image quality using 64-HDCT. Eur Heart J Cardiovasc Imaging. 2013. doi:10.1093/ehjci/jet013.

49. Funama Y, Oda S, Utsunomiya D, et al. Coronary artery stent evaluation by combining iterative reconstruction and high-resolution kernel at coronary CT angiography. Acad Radiol. 2012;19:1324-31.

50. Min JK, Swaminathan RV, Vass M, et al. High-definition multidetector computed tomography for evaluation of coronary artery stents: comparison to standard-definition 64-detector row computed tomography. J Cardiovasc Comput Tomogr. 2009;3: 246-51.

51. Oda S, Utsunomiya D, Funama Y, et al. Improved coronary instent visualization using a combined high-resolution kernel and a hybrid iterative reconstruction technique at 256-slice cardiac CTPilot study. Eur J Radiol. 2013;82:288-95.

52. Do S, Karl WC, Liang Z, et al. A decomposition-based CT reconstruction formulation for reducing blooming artifacts. Phys Med Biol. 2011;56:7109-25.

53. Gebhard C, Fiechter M, Fuchs TA, et al. Coronary artery calcium scoring: influence of adaptive statistical iterative reconstruction using 64-MDCT. Int J Cardiol. 2013;167(6):2932-7.

54. Stenner P, Schmidt B, Allmendinger T, et al. Dynamic iterative beam hardening correction (DIBHC) in myocardial perfusion imaging using contrast-enhanced computed tomography. Invest Radiol. 2010;45:314-23.

55. Boas FE, Fleischmann D. Evaluation of two iterative techniques for reducing metal artifacts in computed tomography. Radiology. 2011;259:894-902.

56. Isola AA, Grass M, Niessen WJ. Fully automatic nonrigid registration-based local motion estimation for motion-corrected iterative cardiac CT reconstruction. Med Phys. 2010;37: 1093-109.

57. Fuchs TA, Fiechter M, Gebhard C, et al. CT coronary angiography: impact of adapted statistical iterative reconstruction (ASIR) on coronary stenosis and plaque composition analysis. Int J Cardiovasc Imaging. 2013;29:719-24. 